\title{
On the Quadrant-Electrometer
}

This content has been downloaded from IOPscience. Please scroll down to see the full text. 1885 Proc. Phys. Soc. London 77

(http://iopscience.iop.org/1478-7814/7/1/303)

View the table of contents for this issue, or go to the journal homepage for more

Download details:

IP Address: 141.211.4.224

This content was downloaded on 06/09/2015 at 19:44

Please note that terms and conditions apply. 


\section{On an Integrating Anemometer. By Walter Baily, M.A.*}

To this anemometer, which had been already described (Phys. Soc. Proc. vol. vi. p. 115), mechanical counters similar to those used for bicycles had been added, as it has been found that the electric counters with which the instrument was originally made were liable to let the batteries run down when the wind dropped, and were therefore only suited for observations of short duration.

\section{On the Quadrant-Electrometer. \\ By J. Hopkinson, D.Sc., F.R.S.†}

IN Professor Clerk Maxwell's 'Electricity' (vol. i. p. 273) it is proved that the deflection of the needle of a quadrantelectrometer varies as $(A-B)\left(C-\frac{A+B}{2}\right)$, where $C$ is the potential of the needle, and $A$ and $B$ of the two pairs of quadrants. Desiring to ascertain the value of the standard charge of my instrument, I endeavoured to do so by the aid of this formula, and also by a more direct method. The results were quite discordant. Setting aside the special reasoning by which the formula is attained, we should confidently expect that the sensibility of a quadrant-electrometer would increase continuously as the charge of the jar is increased, until at last a disruptive discharge occurs. In my instrument this is not the fact. As the charge was steadily increased by means of the replenisher, the deflection of the needle due to three Daniell's elements at first increased, then attained a maximum; and with further increase of charge actually diminished. On turning the replenisher in the inverse direction the sensibility at first increased, attained the maximum previously observed, and only on further reduction of charge diminished.

Before giving the experimental results, it may be worth while to briefly examine the theory of the quadrant-electrometer. Let A, B, C, D be the potentials of the quadrants, the needle,

* Read February 14, 1885.

+ Read March 14, 188.万. 
and the inductor which is used for measuring high potentials (see Reprint of Sir W. Thomson's papers, p. 278). Let $Q_{1}$, $\mathrm{Q}_{2}, \mathrm{Q}_{3}, \mathrm{Q}_{4}$ be the quantities of electricity on these bodies respectively, and $\theta$ the angle of deflection of the needle, measured in terms of divisions of the scale, on which the image of the lamp-flame is projected. We have the equations

$$
\left.\begin{array}{l}
\mathrm{Q}_{1}=q_{11} \mathrm{~A}-q_{12} \mathrm{~B}-q_{13} \mathrm{C}-q_{14} \mathrm{D} \\
\mathrm{Q}_{2}=-q_{12} \mathrm{~A}+q_{22} \mathrm{~B}-q_{23} \mathrm{C}-q_{24} \mathrm{D} \\
\mathrm{Q}_{3}=-q_{13} \mathrm{~A}-q_{23} \mathrm{~B}+q_{33} \mathrm{C}-q_{34} \mathrm{D} \\
\mathrm{Q}_{4}=-q_{14} \mathrm{~A}-q_{24} \mathrm{~B}-q_{34} \mathrm{C}+q_{44} \mathrm{D} .
\end{array}\right\}
$$

$q_{11}$ \&c. are the coefficients of capacity and induction. They are independent of $\mathrm{A}, \mathrm{B}, \mathrm{C}, \mathrm{D}$, and are functions of $\theta$ only. As above written, they are all pasitive. Let the energy of electrification be $\mathrm{W}:-$

$$
\left.\begin{array}{rl}
2 \mathrm{~W} & =q_{11} \mathrm{~A}^{2}+q_{22} \mathrm{~B}^{2}+q_{33} \mathrm{C}^{2}+q_{44} \mathrm{D}^{2} \\
& -2 q_{12} \mathrm{AB}-2 q_{13} \mathrm{AC}-2 q_{14} \mathrm{AD} \\
& -2 q_{23} \mathrm{BC}-2 q_{24} \mathrm{BD} \\
& -2 q_{34} \mathrm{CD}
\end{array}\right\} .
$$

Equations (1) and (2) are perfectly general, true whatever be the form of the four bodies.

If the four quadrants completely surround the needle,

$$
\left.\begin{array}{l}
q_{34}=0, \\
q_{14}, q_{24}, \text { and } q_{44} \text { are independent of } \theta, \\
q_{33}=q_{13}+q_{23}
\end{array}\right\} .
$$

Now when the electrometer is properly adjusted, the needle will not be deflected when $A=B$, whatever $C$ and $A$ may be. Hence $A-B$ is a factor of $\frac{d W}{d \theta}$, and we have

whence

$$
\left.\begin{array}{l}
\frac{d q_{11}}{d \theta}+\frac{d q_{22}}{d \theta}=2 \frac{d q_{12}}{d \theta} \\
\frac{d q_{33}}{d \theta}=0
\end{array}\right\}
$$

$$
2 \frac{d \mathrm{~W}}{d \theta}=(\mathrm{A}-\mathrm{B})\left(\mathrm{A} \frac{d q_{11}}{d \theta}-\mathrm{B} \frac{d q_{22}}{d \theta}-\mathrm{C} \frac{d q_{13}}{d \theta}\right) .
$$

This should be true of any electrometer having the above adjustment correctly made. 
But by suitably forming the three bodies A, B, C, further relations between the coefficients may be obtained. The condition of symmetry would give us $\frac{d q_{11}}{d \theta}=-\frac{d q_{22}}{d \theta}$; but it is not necessary to assume symmetry. If the circumferential termination of the needle be a circle centre in the axis of suspension (at least near the division of the quadrants), if the needle turn in its own plane, if the quadrants are each approximately a surface of revolution about the axis, and if the radial terminations of the needle be not within the electrical influence of the quadrants within which they are not, conditions closely satisfied in Sir W. Thomson's electrometer,

$$
\begin{aligned}
& \frac{d q_{11}}{d \theta}=\frac{1}{2} \frac{d q_{13}}{d \theta} \\
& \frac{d q_{22}}{d \theta}=-\frac{1}{2} \frac{d q_{13}}{d \theta} .
\end{aligned}
$$

If $\theta$ be small, we obtain

$$
2 \frac{d W}{d \theta}=\alpha(A-B)\left(C-\frac{A+B}{2}\right)
$$

the formula in Maxwell.

Returning now to our original equation, we hare

$$
\begin{aligned}
2 \mathrm{~W} & =q_{11} \mathrm{~A}^{2}+q_{22} \mathrm{~B}^{2}+q_{33} \mathrm{C}^{2}+q_{44} \mathrm{D}^{2} \\
& -2 q_{12} \mathrm{AB}-q_{33} \mathrm{AC}-2 q_{14} \mathrm{AD} \\
& -q_{33} \mathrm{BC}-2 q_{24} \mathrm{BD} \\
& +\alpha \theta(\mathrm{A}-\mathrm{B})\left(\mathrm{C}-\frac{\mathrm{A}+\mathrm{B}}{2}\right)
\end{aligned}
$$

involving in all eight constants, $q_{11}$ \&c. being now regarded as representing the values of the coefficients in the zero position.

$$
\left.\begin{array}{l}
\mathrm{Q}_{1}=q_{11} \mathrm{~A}-q_{12} \mathrm{~B}-\frac{1}{2} q_{33} \mathrm{C}-q_{14} \mathrm{D}+\alpha \theta(\mathrm{C}-\mathrm{A}), \\
\mathrm{Q}_{2}=-q_{18} \mathrm{~A}+q_{22} \mathrm{~B}-\frac{1}{2} q_{33} \mathrm{C}-q_{24} \mathrm{D}-\alpha \theta(\mathrm{C}-\mathrm{B}), \\
\mathbf{Q}_{3}=-\frac{1}{2} q_{33} \mathrm{~A}-\frac{1}{2} q_{33} \mathrm{~B}+q_{33} \mathrm{C}+\alpha \theta(\mathrm{A}-\mathrm{B}), \\
\mathbf{Q}_{1}=-q_{14} \mathrm{~A}-q_{24} \mathrm{~B}+q_{44} \mathrm{D} .
\end{array}\right\}
$$

We may now discuss a variety of important particular cases.

(a) B is put to earth; A then is connected to a condenser, capacity $a$, charged to potential V: we want to know V from 
the reading of the electrometer. Here

$$
\begin{aligned}
& a \mathrm{~V}-\frac{1}{2} q_{33} \mathrm{C}=\left(a+q_{11}\right) \mathrm{A}-\frac{1}{2} q_{33} \mathrm{C}+\alpha \theta(\mathrm{C}-\mathrm{A}), \\
& \mathrm{V}=\mathrm{A}+\frac{q_{11}}{a} \mathrm{~A}+\frac{\alpha \theta(\mathrm{C}-\mathrm{A})}{a} .
\end{aligned}
$$

Neglecting A compared with $C$, and assuming

we have

$$
\theta=\lambda(\mathrm{A}-\mathrm{B})\left(\mathrm{C}-\frac{\mathrm{A}+\mathrm{B}}{2}\right),
$$

$$
\mathrm{V}=\mathrm{A}\left\{1+\frac{q_{11}}{a}+\frac{\lambda_{\alpha} \mathrm{C}^{2}}{a}\right\}
$$

The apparent capacity of $\mathrm{A}$ increases with $\mathrm{C}$.

(b) B is again zero. A is connected to a source, but is discomnected and insulated when the deflection of the needle is $\theta^{\prime}$; the final deflection is $\theta$ : required the potential $\mathrm{V}$ of the source.

$$
\begin{aligned}
q_{11} \mathrm{~V} & -\frac{1}{2} q_{33} \mathrm{C}+\alpha \theta^{\prime}(\mathrm{C}-\mathrm{V}) \\
& =q_{11} \mathrm{~A}-\frac{1}{2} q_{33} \mathrm{C}+\alpha \theta(\mathrm{C}-\mathrm{A}), \\
\mathrm{V} & =\mathrm{A}+\frac{\alpha\left(\theta-\theta^{\prime}\right) \mathrm{C}}{q_{11}} \\
& =\mathrm{A}\left\{1+\frac{\alpha \lambda\left(1-\frac{\theta^{\prime}}{\theta}\right) \mathrm{C}^{2}}{q_{11}}\right\} .
\end{aligned}
$$

We may now consider the methods of varying the sensibility of the instrument (see Reprint of Sir W. Thomson's papers, p. 280). The methods dealt with are those of Sir W. Thomson, somewhat generalized.

(c) The quadrant $B$ is connected with an insulated condenser, capacity $b$, whilst $A$ is connected to a source of electricity:-

therefore

$$
\begin{aligned}
& 0=-q_{12} \mathrm{~A}+\left(b+q_{22}\right) \mathrm{B}-\alpha \theta \mathrm{C}, \\
& \theta=\lambda(\mathrm{A}-\mathrm{B}) \mathrm{C}
\end{aligned}
$$

so

$$
\begin{aligned}
& 0=-q_{12} \mathrm{~A}+\left(b+q_{22}\right)\left(\mathrm{A}-\frac{\theta}{\lambda \mathrm{C}}\right)-\alpha \theta \mathrm{C} ; \\
& \mathrm{A}=\theta \frac{\frac{b+q_{22}}{\lambda \mathrm{C}}+a \mathrm{C}}{\left(b+q_{22}\right)-q_{12}} .
\end{aligned}
$$

If $b=0$, we have the first reduced sensibility given by Sir W. Thomson. 
(d) All methods of using the inductor may be treated under one general form. Let the quadrants $A$ and $B$ be connected with insulated condensers, capacities $a$ and $b$; then connect the inductor to a source, potential $\mathrm{V}$;

$$
\begin{aligned}
& \left.\begin{array}{l}
0=\left(q_{11}^{\circ}+a\right) \mathrm{A}-q_{12} \mathrm{~B}-q_{14} \mathrm{~V}+a \theta \mathrm{C}, \\
0=-q_{12} \mathrm{~A}+\left(q_{22}+b\right) \mathrm{B}-q_{24} \mathrm{~V}-a \theta \mathrm{C}, \\
0=\lambda(\mathrm{A}-\mathrm{B}) \mathrm{C}
\end{array}\right\} \\
& \left(+q_{11} q_{22}-q_{12}^{2}+a q_{22}+b q_{11}+a b\right)(\mathrm{A}-\mathrm{B}) \\
& +\left\{q_{14}\left(q_{12}-q_{22}-b\right)-q_{24}\left(q_{12}-q_{11}-a\right)\right\} \mathrm{V} \\
& +\left\{-2 q_{12}+q_{22}+q_{11}+b+a\right\} a \theta \mathrm{C}=0 \text {; }
\end{aligned}
$$

whence we have an expression for $\mathrm{V}$ proportional to $\theta$. By a proper choice of $a$ and $b$, we can make the sensibility as low as we please.

Now the whole of these formulæ rest on the same reasoning as the equation

$$
\theta=\lambda(\mathrm{A}-\mathrm{B})\left(\mathrm{C}-\frac{\mathrm{A}+\mathrm{B}}{2}\right)
$$

I have mentioned that, in my instrument at least, this equation quite fails to represent the facts when $C$ is considerable. It becomes a matter of interest to ascertain when the formula begins to err to a sensible extent. If a constant battery of a large number of elements were available, this would be soon accomplished. I have at present set up only 18 Daniells. I have therefore been content to use the electrometer to ascertain its own charge by the aid of the inductor, using the 18 Daniells as a standard potential. As the charges range as high as 2600 Daniell's elements, the higher numbers can only be regarded as very rough approximations; sufficiently near, however, to indicate the sort of result which would be obtained if more precise methods were used. The first column in the following Table gives the ascertained or estimated charge of the jar of my electrometer in Daniell's elements; the second the deflection in scale-divisions caused by three elements; the third, the coefficient $\lambda$, deduced by the formula $\theta=\lambda \mathrm{AC}$ : this coefficient ought theoretically to be constant. 


\begin{tabular}{|c|c|c|}
\hline I. & II. & III. \\
\hline 72 & 75 & $0 \cdot 35$ \\
112 & 118 & 0.35 \\
136 & 140 & $0 \cdot 35$ \\
178 & 190 & $0 \cdot 35$ \\
238 & 239 & $0 \cdot 34$ \\
303 & 288 & $0 \cdot 32$ \\
383 & 336 & $0 \cdot 30$ \\
512 & 391 & $0 \cdot 26$ \\
616 & 409 & $0 \cdot 22$ \\
813 & 432 & $0 \cdot 18$ \\
1080 & 424 & $0 \cdot 13$ \\
1312 & 402 & $0 \cdot 10$ \\
1728 & 360 & 0.07 \\
2124 & 320 & $0 \cdot 05$ \\
2634 & 296 & $0 \cdot 037$ \\
1704 & 353 & $0 \cdot 07$ \\
1436 & 394 & $0 \cdot 09$ \\
1284 & 412 & $0 \cdot 11$ \\
876 & 436 & $0 \cdot 17$ \\
684 & 427 & $0 \cdot 21$ \\
\hline
\end{tabular}

By connecting the jar and one quadrant to 18 elements and the other quadrant to earth, I obtained 0.356 as the value of $\lambda$, making use of the complete equation

$$
\theta=\lambda(\mathrm{A}-\mathrm{B})\left(\mathrm{C}-\frac{\mathrm{A}+\mathrm{B}}{2}\right)
$$

It will be seen that this equation may be trusted until $\mathrm{C}$ is over 200 Daniell's elements potential, but that when $\mathrm{C}$ exceeds 250 a quite different law rules.

The foregoing was read before the Physical Society a few years ago, but I stopped its publication after the type was set up, because I was not satisfied that my appliances for experiment were satisfactory, or that I could give any satisfactory explanation of the anomaly.

The electrometer had been many times adjusted for various purposes before further experiments were made, so that those which I shall now describe cannot be directly compared with what goes before. The old experiment was first repeated, and the existence of a maximum sensibility again found. On examination, it was found that the needle hung a little low so that it was nearer to the part of the quadrant below it than to that above. It is easy to see that this would produce the anomalous result observed, though there is reason for thinking it is not the sole cause. The effect of the needle being low 
is that it will be on the whole attracted downwards; and so the apparent weight hanging on the fibre-suspension and the consequent tension of the fibres will be increased. The increase of the tension will be as the square of the potential $\mathrm{C}$; and hence the formula for the deflection will be modified to

$$
\theta=\frac{\lambda}{1+k \mathrm{C}^{2}}(\mathrm{~A}-\mathrm{B})\left(\mathrm{C}-\frac{\mathrm{A}+\mathrm{B}}{2}\right),
$$

where $k$ is a constant depending upon the extent to which the position of the needle deviates from its true position of midway between the upper and lower parts of the quadrants. By a proper choice of $k$, the results I previously obtained are found to agree well with this formula.

The electrometer was next adjusted in the following way:The needle was raised by taking up the fibres of the suspension and adjusting them to equal tension in the usual way, and the proportionality of sensibility to charge was tested, the charge being now determined in arbitrary units by discharging the jar of the instrument through a ballistic galvanometer. The operation was repeated until the sensibility, so far as this method of testing goes, was proportional to the charge of the jar over a very long range. It was then found that the needle was slightly above the median position within the quadrants. Increased tension of the fibres from electrical attraction does not therefore account for the whole of the facts, although it does play the principal part. The sensibility of the instrument being now at least approximately proportional to the charge of the jar, I proceeded to determine accurately the potential of the jar when charged to the standard as indicated by the idiostatic gauge.

In what follows the quadrants, one of which is under the induction-plate, are denoted by $B$, the others by $A$. The quadrants $B$ are connected to the case, $A$ are insulated. The jar is connected to the induction-plate, and the reading on the scale noted; the connection is broken, and the inductionplate is connected to the case, and the reading on the scale again noted; the difference is the deflection due to the charge in the jar. It is necessary to read the scale for zero-charge on induction-plate last, because the charging of the induction-plate slightly diminishes the charge of the jar, and considerably displaces the zero-reading by giving an induc- 
tive charge to the quadrant $A$. It is also necessary to begin with the charge of the jar minutely too high, so that after separating the induction-plate from the interior of the jar, the latter shall have exactly the correct charge as indicated by the gauge. The deflection thus obtained was precisely $298 \frac{1}{2}$, repeated in many experiments. The double deflection given by seventy Daniell cells was 43.6 scale-divisions. By comparison with two Clark's cells, the value of which I know, the potential of the seventy Daniells was found to be $74 \cdot 2$ volts; hence the potential of the jar is 1016 volts, when charged to the potential indicated by the gauge.

The constant $\lambda$ of the instrument was next determined by the formula

$$
\theta=\lambda(\mathrm{A}-\mathrm{B})\left(\mathrm{C}-\frac{\mathrm{A}+\mathrm{B}}{2}\right) .
$$

Four modes of connecting are available for this :--

$$
\begin{aligned}
& \mathrm{A}=\mathrm{C}=74 \cdot 2 \text { volts, } \mathrm{B}=0 ; \\
& \mathrm{B}=\mathrm{C}=74 \cdot 2 \text { volts }, \mathrm{A}=0 ; \\
& \mathrm{A}=\mathrm{C}=0, \mathrm{~B}=74 \cdot 2 \text { volts; } \\
& \mathrm{B}=\mathrm{C}=0, \mathrm{~A}=74 \cdot 2 \text { volts. }
\end{aligned}
$$

In each case the deflection was 253.5 if the charge on the needle was positive in relation to the quadrant with which it was not connected; and was 247 when the needle was negative. This at first appeared anomalous; but the explanation is very simple. The ncedle is aluminium, the quadrants are either brass or brass-gilded, I am not sure which. There is therefore a contact-difference of potential between the needle and the quadrants; call it $x$. Thus, instead of $\theta=\frac{A^{2}}{2}$, we have

$$
\theta=+\lambda \mathrm{A}\left(\frac{\mathrm{A}}{2}+x\right)
$$

and

this gives

$$
\theta=\lambda(-\mathrm{A})\left(-\frac{\mathrm{A}}{2}+x\right) \text {; }
$$

$$
x=\frac{6 \cdot 5}{2 \lambda \mathrm{A}}=\mathrm{U} \cdot 482 \text { volt. }
$$

The result was verified by using fourteen cells mstead of seventy: the deflections were 10.0 and 8.8 , which gives the same value to $x$. It is worth noting that the same cause affects the idiostatic gauge in the same way. Let the jar be charged till the gauge comes to the mark. Call $P$ the differ- 
ence of potential between the aluminium lever of the idiostatic gauge and the brass disk below which attracts it. The difference of potential between the brass of the case and the brass work of the interior is $\mathrm{P}+x$, and between the case and the aluminium needle within the quadrants it is $\mathrm{P}+2 x$. If, however, the charge is negative, the difference is $-\mathrm{P}+2 x$. Hence the sensibility will be different from two causes, according as the jar is charged positively or negatively, till the idiostatic gauge is at its standard. For determining the constant $\lambda$ we must take the mean of the two results 253.5 and 247 , that is $250 \cdot 25$. Comparing this with the actual standard charge of the jar, and the double deflection given by one volt $172 \cdot 4$, when charged to the standard, we see that the irregularity has not been wholly eliminated. It appeared desirable to determine the sensibility of the instrument for a lower known charge. The charge was determined exactly as described above, and was found to be 609 volts; whilst 1 volt gave $107 \cdot 1$ scale-divisions double deflection; whence in the equation

we have, if

$$
\theta=\frac{\lambda(\mathrm{A}-\mathrm{B}) \mathrm{C}}{1+k \mathrm{C}^{2}}
$$

$$
\lambda=0 \cdot 1816, k=7 \times 10^{-8},
$$

the following as the calculated and observed deflections:-

$$
\begin{aligned}
& \text { Calculated . . . . } 250 \cdot 0,107 \cdot 7,172 \cdot 4 \\
& \text { Observed . • . 250.2, 107.1, 172.4, }
\end{aligned}
$$

which is well within errors of observation.

This deviation from proportionality of sensibility did not appear to be worth correcting, as I was not sure that other small irregularities might not be introduced by raising the needle above the middle position within the quadrants. It appears probable that the small deviation still remaining does not arise from the attraction of the quadrants on the needle increasing the tension of the suspension, but from some cause of a quite different nature, for if it were so caused the capacityequations would be

where

$$
\theta_{1}=q_{11} \mathrm{~A}-q_{12} \mathrm{~B}-q_{33} \mathrm{C}-g_{14} \mathrm{D}+\alpha \theta \mathrm{C}, \& \mathrm{c},
$$

$$
\theta=\frac{\lambda}{1+k \mathrm{C}^{2}}(\mathrm{~A}-\mathrm{B})\left(\mathrm{C}-\frac{\mathrm{A}+\mathrm{B}}{2}\right)
$$


Now the experiments I have tried for determining $q_{11}, q_{12}$, $\& c$., are not in accord within the limits of errors of observation, using these equations of capacity; but they are in better accord if, in lieu of the term $\alpha \theta \mathrm{C}$, we write $\frac{\alpha \theta \mathrm{C}}{1+k \mathrm{C}^{2}}$. I have no explanation of this to offer; but in what follows it is assumed that the equations expressing the facts are

$$
\begin{aligned}
\theta & =\mu(\mathrm{A}-\mathrm{B}), \text { where } \mu=\frac{\lambda \mathrm{C}}{1+k \mathrm{C}^{2}} \\
\mathrm{Q}_{1} & =q_{11} \mathrm{~A}-q_{12} \mathrm{~B}-q_{14} \mathrm{D}+\beta \mu \theta \\
\mathrm{Q}_{2} & =-q_{12} \mathrm{~A}+q_{22} \mathrm{~B}-q_{24} \mathrm{D}-\beta \mu \theta \\
\mathrm{Q}_{4} & =-q_{14} \mathrm{~A}-q_{24} \mathrm{~B}+q_{44} \mathrm{D}
\end{aligned}
$$

We are now in a position to determine the various coefficients of capacity : in doing so it is necessary to distinguish the values of $q_{11}$ and $q_{22}$ when the posts by which contact with the quadrants is made are down and in contact with the quadrants, and when they are raised up out of contact; the former are denoted by $q_{11}+a$ and $q_{22}+a$, the latter by $q_{11}$ and $q_{22}$, the capacity of the binding-posts being $a$. As a convenient temporary unit of capacity the value of $\beta \mu^{2}$, when the jar has the standard charge, is taken. The first set of experiments was to determine the deflections caused by known potentials with varied charge of jar, one or other of the quadrants being insulated. Three potentials of the jar were used - that of the standard indicated by the idiostatic gauge and two lower. The values of $\mu$ are denoted by $\mu_{3}, \mu_{2}, \mu_{1}$. It was found by connecting the two quadrants to standard cells that

and hence

$$
\mu_{3}: \mu_{2}: \mu_{1}=1: 0.805: 0.585 \text {; }
$$

$$
\beta \mu_{3}^{2}=1, \beta \mu_{2}^{2}=0.648, \beta \mu_{1}^{2}=0.342 \text {. }
$$

Suppose quadrant $A$ be insulated, and potential $B$ be applied to quadrant $B$; then we have, if $\theta$ be deflection which potential $B$ would cause with standard charge, if quadrant $A$ were connected to the case, and $\phi$ the observed deflection,

whence

$$
\begin{aligned}
& 0=q_{11} \mathrm{~A}-q_{12} \mathrm{~B}+\beta \mu \phi ; \\
& \phi=\mu(\mathrm{A}-\mathrm{B}) ; \\
& \theta=-\mu_{3} \mathrm{~B} ;
\end{aligned}
$$

$$
\phi=\theta \cdot \frac{\mu}{\mu_{3}} \cdot \frac{q_{11}-q_{12}}{q_{11}+\beta \mu^{2}}
$$


In the calculated values of $\phi$ given below,

$$
\begin{aligned}
& q_{11}=0.502, \quad q_{22}=0.543 \\
& q_{12}=0.293, \quad a=0.200 \text { for B, } \\
& =0.193 \text { for } A \text {. }
\end{aligned}
$$

A closer approximation to observation is obtained byassuming the two contact-posts to be of slightly different capacities; the difference given above is no more than might be expected to exist.

The jar being charged to standard potential, $B$ was insulated, and its post raised, and A was connected to 10 Daniells, for which $\theta=1808$ :-

$$
\begin{aligned}
\text { Deflection observed } & =293 \cdot 2, \\
, \quad \text { calculated } & =293 \cdot 0 .
\end{aligned}
$$

The post of $\mathrm{B}$ was lowered to contact :-

$$
\begin{aligned}
\text { Deflection observed } & =467 \cdot 0, \\
, \quad \text { calculated } & =466 \cdot 8 .
\end{aligned}
$$

A was now insulated and post raised, $B$ was connected to the same battery:-

$$
\begin{aligned}
\text { Deflection observed } & =251 \cdot 0, \\
, \quad \text { calculated } & =251 \cdot 6 .
\end{aligned}
$$

The post of A was lowered to contact :-

$$
\begin{aligned}
\text { Deflection observed } & =429 \cdot 0, \\
, \quad \text { calculated } & =428 \cdot 8 .
\end{aligned}
$$

The jar was now charged to a lower potential, for which $\mu=\mu_{2}$ with $\mathrm{B}$ insulated and post raised, and $\mathrm{A}$ connected to 30 Daniells, for which $\theta=5468$ :-

$$
\begin{aligned}
\text { Deflection observed } & =925 \cdot 0, \\
, \quad \text { calculated } & =924 \cdot 0 .
\end{aligned}
$$

The post of $B$ was lowered to contact, and $A$ connected to 10 Daniells, for which $\theta=1808$ :-

$$
\begin{aligned}
\text { Deflection observed } & =470 \cdot 5, \\
, \quad \text { calculated } & =470 \cdot 85 .
\end{aligned}
$$

A was now insulated and post raised, $B$ was connected toVOL. VII. 
a battery of 30 Daniells, for which $\theta=5468$ :-

Deflection observed $=798.0$,

, calculated $=800^{\circ} 0$.

The post of $A$ was lowered to contact, and $B$ was connected to 10 Dauiells ; $\theta=1808$ :-

Deflection olserved $=437 \cdot 0$, ,$\quad$ calculated $=435 \cdot 7$.

The jar was then charged to a still lower potential, for which $\mu=\mu_{1}$, with $B$ insulated and post raised, and $A$ connected to 30 Daniells, for which $\theta=5468:-$

Deflection ohserved $=901 \cdot 0$, " calculated $=903 \cdot 6$.

The post of $B$ was lowered to contact and $A$ connected to 10 Daniells ; $\theta=1808$ :-

$$
\begin{aligned}
\text { Deflection observed } & =437 \cdot 0, \\
, \quad \text { calculated } & =438 \cdot 7 .
\end{aligned}
$$

A was now insulated and post raised, and $B$ was connected to 30 Daniells ; $\theta=5468$ :-

Deflection observed $=785$,

, calculated $=7,2$.

The post of $A$ was lowered to contact and $B$ connected to 10 Daniells ; $\theta=1808$ :-

$$
\begin{aligned}
\text { Deflection observed } & =408, \\
, \quad \text { calculated } & =410 .
\end{aligned}
$$

The next experiment was similar, excepting only that the insulated quatrunt $B$ was connected to a condenser; this condenser consisted merely of a brass tuhe insulated within a larger tube-its capacity is about $0 \cdot 00000$ microfarad. The jar was at its standard charge. Calling the capacity of the condenser $l$, in terms of our temporary unit, we have, as before,

$$
\phi=\theta \frac{\vec{b}+a+q_{22}-q_{12}}{b+u+q_{22}+1} \quad\left[\beta \mu_{3}=1\right] .
$$

When $\theta=1259, \phi$ was observed to be 427 , whence $7=3.950$.

We are now in a position to obtain inderendent voritioa-

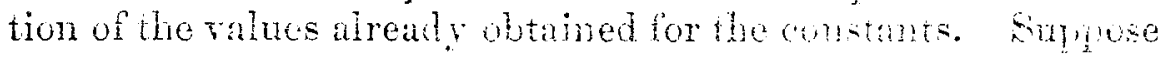
A be comected to the case, that condenser $t$ is charged from 
a battery of known potential, such that it would give deflection $\theta$ if connecter to $B$, and the charged condenser is then connected to B. Suppose $\psi$ be the deflection before connection is made, $\phi$ after. Then

$$
l(\theta-\phi)=\left\{q_{22}+a+1\right\}(\phi-\psi) \text {. }
$$

When $\theta=1439$ and $\psi=0$, it was found that $\phi=915$. The value of $\phi$, calculated from the ralues of the constants already obtained, is 928 .

When $\theta=1439$ and $\psi=-676$, it was found that $\phi=+676$; the calculated ralue is 688 .

A further experinent of verification, involving only the capacity of the qualrant, is the following. The quadrant $A$ being connected to the case, $B$ was charged by contact instantaneously mado and broken with a battery of known potential, and the resulting deflection was noted. The instantaneous contact being made by hand, no very great accuracy could be expected. Let $\psi$ and $\phi$ be the readings on the scale before and after the instantaneous contact; then

$$
\frac{\theta-\phi}{\phi-\psi}=\frac{1}{q_{22}+a}=1 \cdot 345 \text {. }
$$

The following results were obtained:-

$\theta$.

1796

1796 $\psi$.

0

$-493$ $\phi$ observed. $\phi$ calculated.

763

493
765

482

We noxt determine the coefficients $q_{14}$ and $q_{24}$ of induction of the induction-plate on the quadrants. This is easily done from the deflections obtained with the induction-plate, one or both pairs of quadrants being insulated. First, suppose one pair, say $B$, are insulated whilst $A$ is connected to the case:-

$$
\begin{aligned}
& 0=+\varphi_{22} B-q_{21} D-\beta \mu \phi, \\
& \phi=-\mu \mathrm{B}, \\
& \theta=\mu_{3} D ;
\end{aligned}
$$

whenco

$$
\phi=\theta_{\mu_{3}}^{\mu} \frac{q_{24}}{q_{22}+\beta_{\mu}^{2}}
$$

$\Phi$ being the deflection actually observed, and $\theta$ that which 
the battery used would give if connected direct to the quadrants, the needle having the standard charge. When $\theta$ was 12,800 and $\mu=\mu_{3}, \phi$ was 418 , whence $q_{24}=0.0504$.

In the same way $A$ being insulated but $B$ connected to the case, $\phi$ was found to be $43 \cdot 6$, whence $q_{14}=0 \cdot 00508$.

Again, when both quadrants are insulated we have

$$
\begin{aligned}
& 0=q_{11} \mathrm{~A}-q_{12} \mathrm{~B}-q_{14} \mathrm{D}+\beta \mu \phi, \\
& 0=-q_{12} \mathrm{~A}+q_{22} \mathrm{~B}-q_{24} \mathrm{D}-\beta \mu \phi, \\
& \phi=\mu(\mathrm{A}-\mathrm{B}), \\
& \theta=\mu_{3} \mathrm{D} .
\end{aligned}
$$

From the first two equations,

whence

$$
\begin{gathered}
\left(q_{11} q_{22}-q_{32}^{2}\right)(\mathrm{A}-\mathrm{B})-\left\{\left(q_{22}-q_{12}\right) q_{14}-\left(q_{11}-q_{12}\right) q_{24}\right\} \mathrm{D} \\
+\left(q_{22}+q_{11}-2 q_{12}\right) \beta \mu \phi=0
\end{gathered}
$$

$$
\phi=\theta \frac{\mu}{\mu_{3}} \frac{\left(q_{22}-q_{12}\right) q_{14}-\left(q_{11}-q_{12}\right) q_{24}}{\left(q_{11} q_{22}-q_{12}^{2}\right)+\left(q_{22}+q_{11}-2 q_{12}\right) \overline{\beta \mu}^{2}} .
$$

In the case when $\mu=\mu_{3}$, substituting the values already determined, we have

$$
\phi=\theta \times 0.0142
$$

it was observed with $\theta=12,800$ that $\phi=183$; the calculated value would be 182 .

With a lower charge on the jar, viz. when $\mu=\mu_{3} \times 0.805$, with $B$ insulated, A connected to the case, and $\theta=12,800$, it was found that $\phi=437.5$; the calculated value is 441 .

The capacity $q_{44}$ of the induction-plate is of no use; its value, however, is about 0.004 , in the same unit as has been so far used.

The capacity $q_{33}$ of the needle and the coefficient of induction of the needle on either quadrant $\frac{1}{2} q_{33}$ are also of no ase, but the method by which they may be obtained is worth noting. Let quadrants $A$ be connected to the case, and let B be insulated, diminish the charge of the jar slightly by the replenisher, and suppose the consequent deflection be $\phi$. Let $\mu$ and $\mu^{\prime}$ be the values of $\mu$ before and after the diminution of charge, as ascertained by applying a known potential- 
difference between the two pairs of quadrants; we have

$$
-q_{33} \mathrm{C}=+q_{22} \mathrm{~B}-q_{33} \mathrm{C}^{\prime}-\beta \mu^{\prime} \phi,
$$

where

$$
\phi=-\mu^{\prime} \mathrm{B} \text {, }
$$

$$
q_{33}\left(\mathrm{C}-\mathrm{C}^{\prime}\right)=\left(q_{22}+\beta \mu^{\prime 2}\right) \mathrm{B},
$$

which determine $q_{33}$, since $\mathrm{C}$ and $\mathrm{C}^{\prime}$ are known from $\mu$ and $\mu^{\prime}$.

Of course the values of the constants of an electrometer are of no value for any instrument except that for which they are determined in the state of adjustment at the time. For any particular use of the instrument it is best to determine exactly that combination of constants which will be needed. Nor is there anything new in principle in the discussion or experiments here given; they are merely for the most part the application of well-known principles to methods of using the electrometer given by Sir William Thomson himself. The method of determining the capacity of a condenser by charging it and connecting it to an insulated quadrant has been used by Boltzmann. But the invention of the quadrant-electrometer by Sir W. Thomson may be said to have marked an epoch in Electrostatics, and the instrument from time to time finds new uses. It therefore seems well worth while to make known observations made upon it in which the instrument itself has been the only object studied. Some practical conclusions may, however, be drawn from the preceding experiments. Before using the formula

$$
\theta=\lambda(\mathrm{A}-\mathrm{B})\left(\mathrm{C}-\frac{\mathrm{A}+\mathrm{B}}{2}\right)
$$

it is necessary to verify that it is sufficiently nearly true, or to determine its variation from accuracy. Unless it be sufficiently accurate through the range experimented upon, the electrometer cannot be applied by the methods well known for determining alternating potentials and the work done by alternating currents.

My pupil, Mr. Paul Dimier, has very efficiently helped me in the execution of the experiments of verification. 